\title{
Verzeichnis der abgekürzt zitierten Literatur
}

Soweit andere als im nachfolgenden Verzeichnis angegebene Auflagen zitiert werden, sind diese mit einer hochgestellten Ziffer gekennzeichnet.

ADS
Altmeppen
Baetge/Kirsch/Thiele/
Bearbeiter
Baumbach/Hueck/Bearbeiter
GmbHG
Baumbach/Hopt/Bearbeiter
Beck HdR/Bearbeiter
BeckOK-HGB/Bearbeiter
Beck BilKomm-Bearbeiter
Boos/Fischer/Schulte-Mattler/
Bearbeiter KWG

Ebenroth/Boujong/Joost/ Strohn/Bearbeiter; EBJS

Emmerich/Habersack KonzernR

Ensthaler
Geßler/Hefermehl/Eckardt/ Kropff
GroßkommAktG/Bearbeiter Großkomm/Bearbeiter

\author{
Habersack/Verse \\ Hachmeister/Kahle/Mock/ \\ Schüppen/Bearbeiter \\ HdR-EA/Bearbeiter \\ Hüffer/Koch AktG \\ KölnKomm-AktG/Bearbeiter \\ KölnKomm-RLR/Bearbeiter
}

Lutter/Hommelhoff/Bearbeiter $\mathrm{GmbHG}$

MünchKommAktG/Bearbeiter
Adler/Düring/Schmaltz (Hrsg.), Rechnungslegung und Prüfung der Unternehmen, Stuttgart, 6. Aufl. 1995-2000

Roth/Altmeppen, GmbHG-Gesetz betreffend die Gesellschaften mit beschränkter Haftung, Kommentar, München, 10. Aufl. 2021

Baetge/Kirsch/Thiele (Hrsg.) Bilanzrecht, Bonn/Berlin, 92. Ergänzungslieferung Juni 2020 (Loseblatt)

Baumbach/Hueck, GmbH-Gesetz, München, 22. Aufl. 2019

Baumbach/Hopt, Handelsgesetzbuch, München, 40. Aufl. 2021

Böcking/Gros/Oser/Scheffler/Thormann (Hrsg.), Beck'sches Handbuch der Rechnungslegung, München 62. Aufl. 2020 (Loseblatt)

Häublein/Hoffmann-Theinert (Hrsg.), Beck'scher Online Kommentar zum HGB, 31. Ed. Stand 15.1.2021

Grottel/Schmidt/Schubert/Störk (Hrsg.), Beck'scher Bilanz-Kommentar, München, 12. Aufl. 2020

Boos/Fischer/Schulte-Mattler (Hrsg.), KWG, CRR-VO: Kommentar zu Kreditwesengesetz, VO (EU) Nr. 575/2013 (CRR) und Ausführungsvorschriften, 2 Bd., 5. Aufl. 2016

Ebenroth/Boujong/Joost/Strohn (Hrsg.), Handelsgesetzbuch: HGB, München, 1. und 2. Bd., 3. Aufl. 2014 f, 4. Aufl. 2020

Emmerich/Habersack, Aktien- und GmbH-Konzernrecht Kommentar, München 9. Aufl. 2019

Ensthaler (Hrsg.), Gemeinschaftskommentar zum Handelsgesetzbuch: HGB, 8. Aufl. 2015

Geßler/Hefermehl/Eckardt/Kropff, Aktiengesetz, $1973 \mathrm{ff}$

Hirte/Mülbert/Roth (Hrsg.), Aktiengesetz Großkommentar, Berlin, 5. Aufl. $2015 \mathrm{ff}$ Staub, Handelsgesetzbuch: Großkommentar, Berlin, 5. Aufl. 2008 ff, 6. Aufl. $2021 \mathrm{ff}$

Habersack/Verse, Europäisches Gesellschaftsrecht, München, 5. Aufl. 2019 Hachmeister/Kahle/Mock/Schüppen (Hrsg.), Bilanzrecht Kommentar, Handelsbilanz - Steuerbilanz - Prüfung - Offenlegung - Gesellschaftsrecht, Köln, 2. Aufl. 2020

Küting/Weber (Hrsg.), Handbuch der Rechnungslegung - Einzelabschluss, Stuttgart, 28. Ergänzungslieferung Juni 2019 (Loseblatt) Hüffer/Koch, Aktiengesetz, München,14. Aufl. 2020

Zöllner/Noack (Hrsg.), Kölner Kommentar zum Aktiengesetz, Band 6, Köln, 3. Aufl. 2004

Claussen/Scherrer (Hrsg.), Kölner Kommentar zum Rechnungslegungsrecht, 1. Aufl. 2010

Lutter/Hommelhoff u. a., GmbH-Gesetz, Köln, 20. Aufl. 2020

Goette/Habersack (Hrsg.), Münchener Kommentar zum Aktiengesetz, München, 4. Aufl. $2012 \mathrm{ff}, 5$. Aufl. $2019 \mathrm{ff}$ 
Verzeichnis der abgekürzt zitierten Literatur

MünchKommBGB/Bearbeiter

MünchKommBilR/Bearbeiter

MünchKommHGB/Bearbeiter

Scholz/Bearbeiter GmbHG

Spindler/Stilz/Bearbeiter AktG Staub ADHGB

Staub/Bearbeiter

Wiedemann/Böcking/Gros/ Bearbeiter BilR
Säcker/Rixecker/Oetker/Limperg (Hrsg. der 8. Aufl.), Münchener Kommentar zum Bürgerlichen Gesetzbuch, München, 7. Aufl. 2015 ff, 8. Aufl. $2018 \mathrm{ff}$ Hennrichs/Kleindiek/Watrin (Hrsg.), Münchener Kommentar zum Bilanzrecht, Band 1 IFRS, München September 2014 (Loseblatt)

Schmidt, Karsten (Hrsg.), Münchener Kommentar zum Handelsgesetzbuch: HGB, München, 4. Aufl. $2016 \mathrm{ff}$

Scholz (Hrsg.), Kommentar zum GmbHG, 3 Bd., Köln, 11. Aufl. 2013 ff, 12. Aufl. $2017 \mathrm{ff}$

Spindler/Stilz (Hrsg.), Aktiengesetz, Kommentar, 2 Bd., München, 4. Aufl. 2019 Staub, Kommentar zum Allgemeinen Deutschen Handelsgesetzbuch, Berlin, 5. Aufl. 1897

Canaris/Habersack/Schäfer (Hrsg.), Staub, Großkommentar zum Handelsgesetzbuch, HGB, Berlin, 5. Aufl. 2008 ff, 6. Aufl. $2021 \mathrm{ff}$

Wiedmann/Böcking/Gros (Hrsg.), Bilanzrecht §§ 238-342e HGB, §§ 135-138, 158-161 KAGB Kommentar, München, 4. Aufl. 2019 